\title{
Continuation of bevacizumab and addition of hormone therapy following weekly paclitaxel therapy in HER2-negative metastatic breast cancer
}

\author{
Andrés Redondol,* \\ Virginia Martínez ${ }^{1, *}$ \\ Pilar Zamora' \\ Beatriz Castelo' \\ Alvaro Pinto' \\ Patricia Cruz' \\ Oliver Higuera' \\ Marta Mendiola ${ }^{2}$ \\ David Hardisson ${ }^{3}$ \\ Enrique Espinosa' \\ 'Medical Oncology Department, \\ ${ }^{2}$ Translational Oncology and \\ Molecular Pathology Laboratory, \\ ${ }^{3}$ Pathology Department, University \\ Hospital La Paz (IdiPAZ), Madrid, \\ Spain \\ *These authors contributed equally \\ to this work
}

This article was published in the following Dove Press journal:

OncoTargets and Therapy

27 November 2014

Number of times this article has been viewed

\begin{abstract}
Background: Bevacizumab plus taxane chemotherapy improves progression-free survival (PFS) versus taxane monotherapy in the first-line treatment of HER2-negative metastatic breast cancer (MBC) and appears promising in the second-line setting. This retrospective analysis evaluated the efficacy and safety of this combination in a real-world setting.
\end{abstract}

Patients and methods: Eligible patients received bevacizumab $(10 \mathrm{mg} / \mathrm{kg}$ days 1 and 15 , every 28 days) plus paclitaxel ( $80 \mathrm{mg} / \mathrm{m}^{2}$ days 1,8 , and 15 , every 28 days) as first-line therapy for $\mathrm{MBC}$, or as subsequent lines, including bevacizumab continuation therapy, at La Paz University Hospital between August 2007 and October 2012. End points included objective response rate (ORR), PFS, overall survival (OS), and safety.

Results: Seventy-eight patients were included. Median PFS was 12.8 months for patients receiving first-line treatment and 9.3 months for subsequent lines. Forty-five patients $(57.7 \%)$ continued bevacizumab after stopping paclitaxel, and had significantly longer PFS and OS than those who did not (hazard ratio [HR] 0.40, 95\% confidence interval [CI] 0.248-0.653, $P<0.001$; HR $0.39,95 \%$ CI $0.218-0.710, P=0.002$; respectively). In the continuation phase, estrogen receptor-positive patients had longer PFS and OS when receiving hormone therapy plus bevacizumab versus patients receiving only bevacizumab (HR 0.50, 95\% CI 0.24-1.04, $P=0.06$; HR $0.43,95 \%$ CI $0.16-1.16, P=0.09$; respectively). Thirty-five patients (44.9\%) reported grade $3-4$ adverse events.

Conclusion: Bevacizumab plus paclitaxel was effective in HER2-negative MBC. Continuation of bevacizumab and addition of hormone therapy following paclitaxel therapy could be beneficial.

Keywords: bevacizumab, first line, maintenance, advanced breast cancer, hormone therapy

\section{Introduction}

Treatment regimens involving the combination of antiangiogenic agents, such as bevacizumab with cytotoxic chemotherapy, have been extensively investigated in metastatic breast cancer (MBC) ${ }^{1-3}$ As highly proliferative tumors, such as those with triple-negative histology, have enhanced angiogenesis and increased vascular endothelial growth factor levels, ${ }^{4,5}$ the addition of bevacizumab to chemotherapy was a rational therapeutic approach in $\mathrm{MBC}$.

The combination of bevacizumab with a taxane significantly improved progressionfree survival (PFS) compared with taxane monotherapy in the first-line treatment of human epidermal growth factor receptor 2 (HER2)-negative MBC in a number of randomized, Phase III studies. In the pivotal E2100 study, median PFS was 11.8 months versus 5.9 months, respectively, for bevacizumab plus paclitaxel versus paclitaxel alone

\footnotetext{
Correspondence: Andrés Redondo Medical Oncology Department, University Hospital La Paz (IdiPAZ),

26I Paseo de la Castellana,

Madrid 28046, Spain

$\mathrm{Tel}+34917277516$

Fax +34 9l 3585204

Email andres.redondos@uam.es
} 
(hazard ratio $[\mathrm{HR}] 0.60, P<0.001) .{ }^{1}$ In addition, the AVADO study reported median PFS of 10.1 months versus 8.2 months for bevacizumab plus docetaxel versus docetaxel plus placebo, respectively (HR 0.77, $P=0.006) .{ }^{2}$ Median PFS was increased from 8.0 months to 9.2 months (HR 0.64, $P<0.001$ ) with the combination of bevacizumab and taxanes versus taxanes alone in the RIBBON-1 study. ${ }^{3}$ However, it should be noted that although these studies in the first-line setting demonstrated a significant PFS benefit with the addition of bevacizumab to taxane chemotherapy, a significant overall survival (OS) benefit was not shown, possibly owing to the confounding effect of postprogression therapy, lack of power of the trials, or treatment crossover during the studies. ${ }^{6}$

Limited data are available on the use of bevacizumab and chemotherapy in subsequent lines of treatment in patients with HER2-negative MBC. However, in the second-line setting, the RIBBON-2 trial demonstrated a PFS benefit for the combination of bevacizumab with chemotherapy versus chemotherapy alone (median PFS 7.2 versus 5.1 months, respectively, HR 0.78; $P=0.0072) .^{7}$ The aim of the current analysis was to determine the efficacy and long-term safety of weekly paclitaxel plus bevacizumab administered as different lines of treatment in patients with HER2-negative MBC in a real-world hospital setting.

\section{Patients and methods}

\section{Study design}

This was a retrospective analysis of patients with HER2negative $\mathrm{MBC}$ who received treatment with bevacizumab (Fritz Hoffmann-La Roche, Basel, Switzerland; $10 \mathrm{mg} / \mathrm{kg}$ on days 1 and 15, every 28 days) plus paclitaxel (Bristol-Myers Squibb, New York, NY, USA; $80 \mathrm{mg} / \mathrm{m}^{2}$ on days 1, 8, and 15, every 28 days) as first-line therapy, second-line therapy, or subsequent lines, including those who received bevacizumab continuation therapy after completion of paclitaxel, at La Paz University Hospital between August 2007 and October 2012. All analyses were approved by the local ethics committee.

\section{Patients}

All patients who received bevacizumab plus paclitaxel in any line of therapy between August 2007 and October 2012 were evaluated. Patients were required to have sufficient follow-up time following treatment initiation (approximately 3 months) to be eligible for response evaluation.

\section{Assessments}

Baseline characteristics were collected for each patient, including disease-free interval (DFI), number of previous therapies, disease grade, and hormone-receptor status. PFS, OS, objective response rate (ORR; defined as the percentage of patients achieving a complete response [CR] or partial response $[\mathrm{PR}]$ ) and clinical benefit (defined as the percentage of patients achieving a CR, PR, or stable disease for $\geq 6$ months) were used to assess efficacy. Adverse events (AEs) were graded according to National Cancer Institute Common Toxicity Criteria version 4.0.

\section{Statistics}

Statistical significance was set at $P=0.05$. PFS and OS were analyzed using Kaplan-Meier methodology, and the log-rank test was used to compare differences in PFS and OS between the subgroups evaluated. A Cox proportional-hazard model was used to estimate the HRs and 95\% confidence intervals (CIs). If overall medians could not be calculated because of insufficient PFS or OS events, a median would be calculated based on the events up to 60 months, with the remaining patients censored. ORR was determined by Response Evaluation Criteria in Solid Tumors (RECIST) version 1.1. Statistical analysis was performed using IBM SPSS Statistics version 21 software.

\section{Results \\ Patients}

Data were analyzed from 78 patients. The median age was 54 years (range 33-76 years), and the majority of patients had hormone receptor-positive disease (Table 1). In total, eleven patients $(14.1 \%)$ had triple-negative breast cancer (TNBC). Most patients (83.3\%) had ductal infiltration, more than half (56.9\%) had grade 3 disease, and the majority (75.6\%) had a DFI $>24$ months or stage IV disease at diagnosis. Overall, 43 patients $(51.1 \%)$ received bevacizumab plus paclitaxel as first-line therapy for HER2-negative MBC. The median treatment duration was 8.2 months (95\% CI 0.56-44.62).

A total of 45 patients $(57.7 \%)$ received bevacizumab as continuation therapy after a variable number of cycles of paclitaxel plus bevacizumab. Of these, 40 patients $(88.9 \%)$ had hormone receptor-positive disease, and 20 of these patients $(44.4 \%)$ received hormone treatment plus bevacizumab during continuation therapy.

\section{Efficacy}

Of the 78 patients analyzed, nine achieved a CR and 33 achieved a PR, giving an ORR of 53.8\% (Table 2). Clinical benefit was seen in 64 patients (82.1\%). Median PFS was 12.8 months for patients receiving bevacizumab as first-line treatment and 9.3 months for those receiving 
Table I Baseline characteristics $(n=78)$

\begin{tabular}{ll}
\hline Characteristic & Patients, $\mathbf{n}(\%)$ \\
\hline Histological subtype & \\
Ductal infiltration & $65(83.3)$ \\
Lobular infiltration & $9(11.5)$ \\
Others & $4(5.1)$ \\
Hormone-receptor status & \\
ER-positive & $67(85.9)$ \\
ER- and PgR-positive & $57(73.1)$ \\
TNBC & II (I4.1) \\
Grade (n=58) & \\
I & $7(12.1)$ \\
2 & $18(31.0)$ \\
3 & $33(56.9)$ \\
Number of metastatic sites & \\
$<3$ & $33(42.3)$ \\
$\geq 3$ or liver metastases & $45(57.7)$ \\
Previous treatment (adjuvant or metastatic setting) & \\
No anthracyclines or taxanes & $21(26.9)$ \\
Anthracyclines & $18(23.1)$ \\
Anthracyclines and taxanes & $39(50.0)$ \\
Number of previous treatment lines for MBC & \\
0 & $43(55.1)$ \\
$\geq$ I & $35(44.9)$ \\
\hline
\end{tabular}

Abbreviations: ER, estrogen receptor; MBC, metastatic breast cancer; PgR, progesterone receptor; TNBC, triple-negative breast cancer.

bevacizumab in second or subsequent lines. Median OS was 39.0 months and 20.4 months, respectively. There were no significant differences in PFS or OS between patients with TNBC or hormone receptor-positive disease between any lines of treatment (median PFS 10.3 versus 8.3 months, $P=0.242$; and median OS 25.7 versus 21.6 months, $P=0.991$ ) or in PFS $(P=0.247)$ or OS $(P=0.175)$ among patients with grade 1, 2, or 3 disease.

Patients who had not previously received taxanes had longer PFS than those who had received prior taxane therapy (13.7 versus 8.3 months, HR $0.63,95 \%$ CI $0.39-1.02$; $P=0.062$; Figure 1A), with no significant difference in OS $(P=0.377)$. Previous treatment with anthracyclines had no influence on PFS $(P=0.241)$ or OS $(P=0.09)$. A significant difference in PFS $(P=0.005)$ but not $\mathrm{OS}(P=0.783)$ was seen between patients with a DFI $\leq 24$ months versus $>24$ months or stage IV disease at diagnosis. Median PFS was

Table 2 Tumor response $(n=78)$

\begin{tabular}{ll}
\hline Response & Patients, n (\%) \\
\hline Overall response rate & $42(53.8)$ \\
Complete response & $9(11.5)$ \\
Partial response & $33(42.3)$ \\
Stable disease & $22(28.2)$ \\
Progressive disease & $13(16.7)$ \\
Unevaluable & $\mathrm{I}(1.3)$ \\
\hline
\end{tabular}
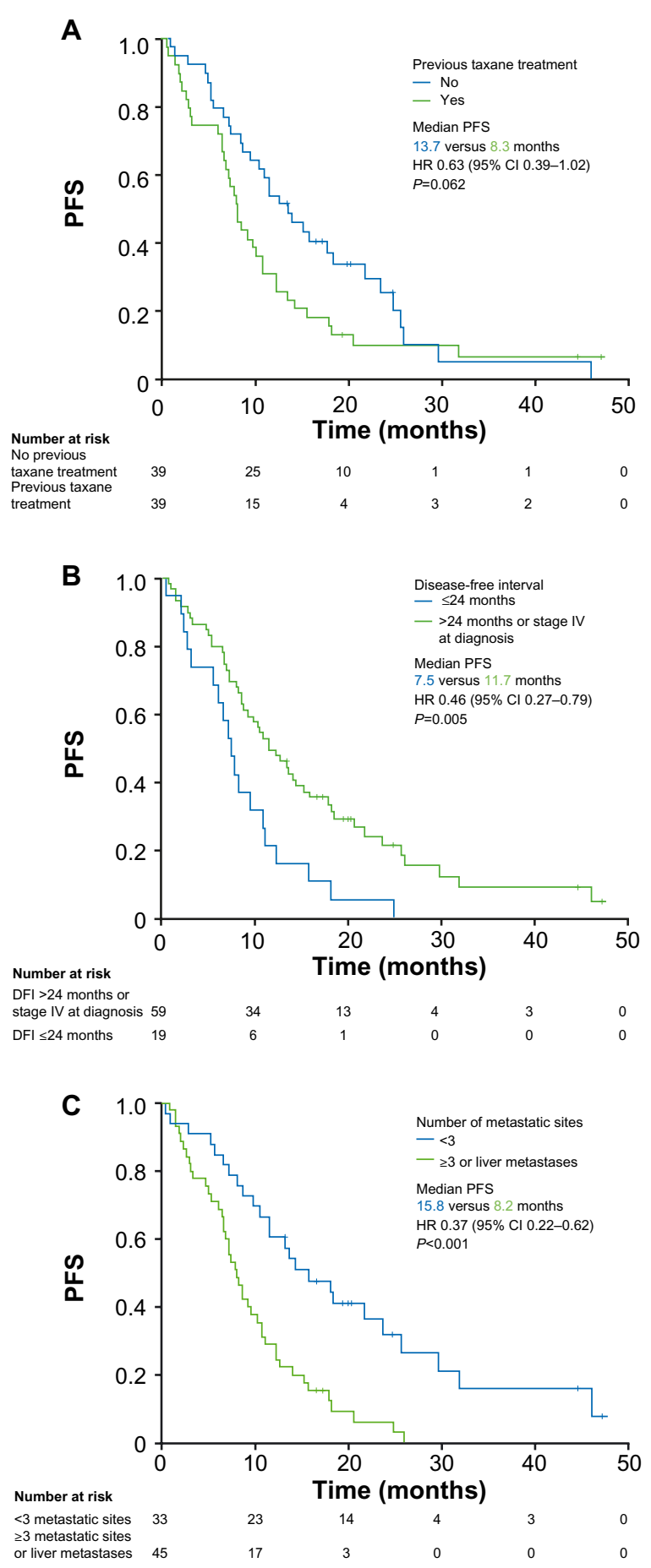

Figure I PFS stratified by (A) previous taxanes (no versus yes), (B) DFI ( $\leq 24$ months versus $>24$ months or stage IV at diagnosis), and (C) number of metastatic sites ( $<3$ versus $\geq 3$ or liver metastases).

Abbreviations: $\mathrm{Cl}$, confidence interval; $\mathrm{DFI}$, disease-free interval; $\mathrm{HR}$, hazard ratio; PFS, progression-free survival.

7.5 months versus 11.7 months, respectively (HR 0.46, 95\% CI 0.27-0.79) (Figure 1B). Patients with fewer than three metastatic sites had a significantly longer median PFS of 15.8 months compared with 8.2 months for patients with three or more metastatic sites or liver metastases (HR 0.37, 
95\% CI $0.22-0.62 ; P<0.001$ ) (Figure $1 \mathrm{C}$ ); there was no significant difference in $\mathrm{OS}(P=0.55)$.

Patients who received bevacizumab as continuation therapy following completion of paclitaxel had a significantly longer PFS than those who did not (median 13.7 versus 5.4 months, HR $0.40,95 \%$ CI $0.25-0.65$; $P<0.001$ ) as well as a significantly longer OS (median 37.4 versus 13.9 months, HR 0.39 , 95\% CI $0.22-0.71$; $P=0.002$ ) (Figure 2). In the subgroup of patients with estrogen receptor-positive disease receiving bevacizumab continuation therapy $(\mathrm{n}=40)$, median PFS was 21.9 months for patients who received hormone treatment plus bevacizumab compared with 10.6 months for those who did not receive hormone treatment (HR $0.50,95 \%$ CI $0.24-1.04$; $P=0.065$ ) (Figure 3A). Median OS was not yet reached in patients receiving concurrent hormone treatment versus 25.7 months for those without hormone treatment (HR 0.43, 95\% CI 0.16-1.16; $P=0.093$ ) (Figure 3B).
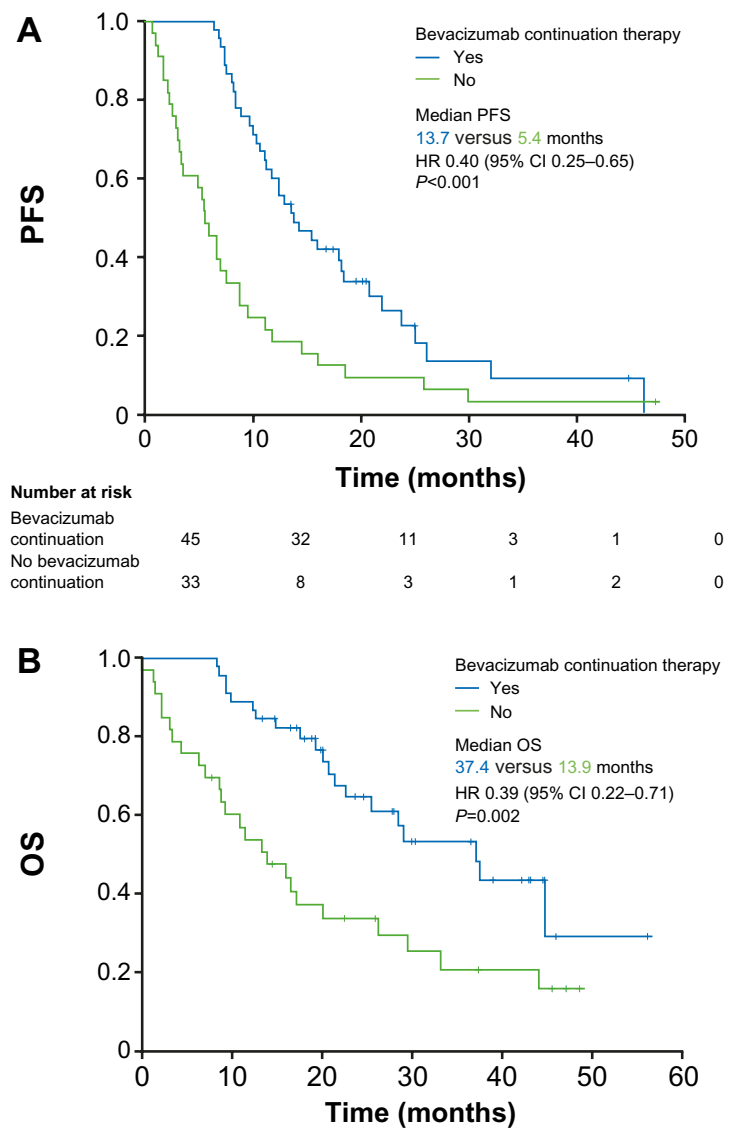

Number at risk

Bevacizumab

$\begin{array}{lllllllll}\text { continuation } & 45 & 40 & 27 & 14 & 8 & 1 & 0 \\ \text { No bevacizumab } & & & & \end{array}$

$\begin{array}{llllllll}\text { continuation } & 33 & 19 & 11 & 6 & 4 & 0 & 0\end{array}$

Figure 2 PFS (A) and OS (B) stratified by continuation therapy (bevacizumab versus no bevacizumab).

Abbreviations: $\mathrm{Cl}$, confidence interval; $\mathrm{HR}$, hazard ratio; OS, overall survival; PFS, progression-free survival.

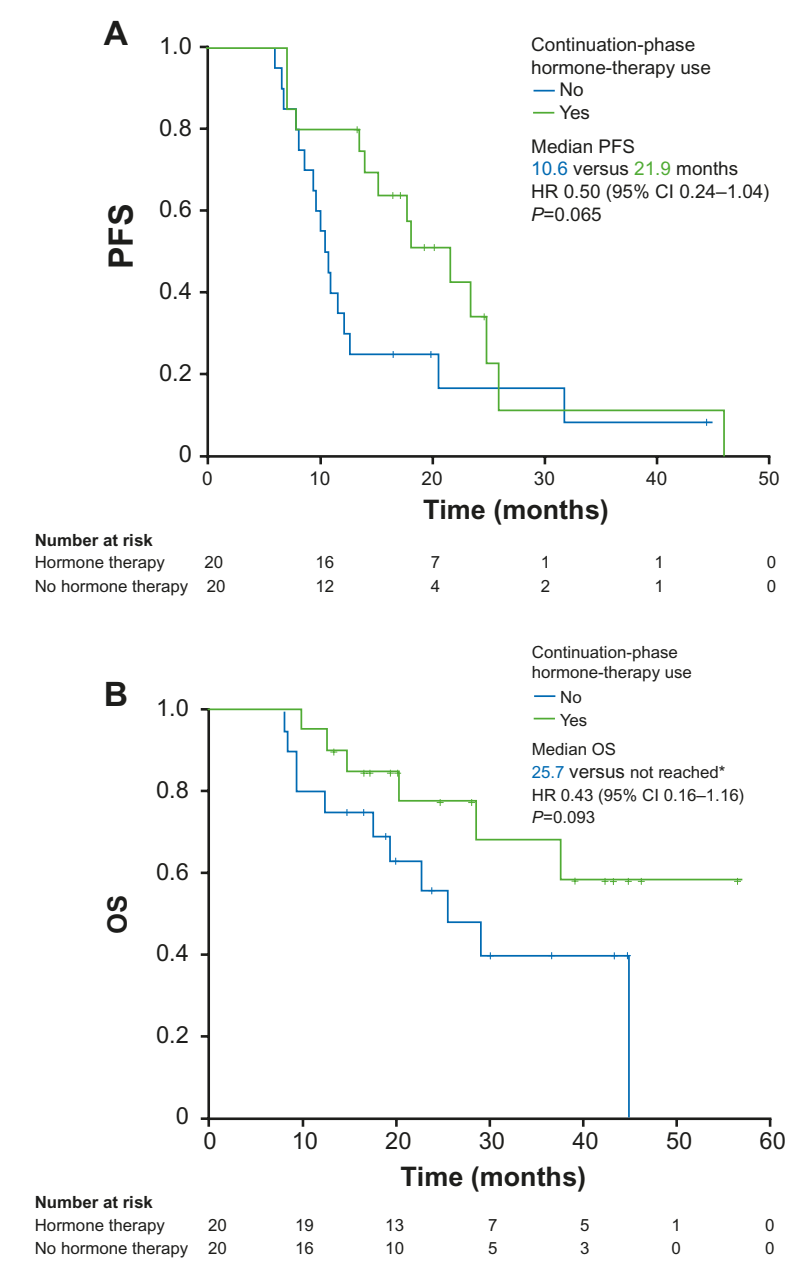

Figure 3 PFS (A) and OS (B) stratified by hormone therapy use in the continuation treatment phase (yes versus no) in the subgroup with estrogen receptor-positive disease.

Notes: *In the hormone-therapy group, the OS median was not reached (immature data); 56.3 months was the maximum value recorded in this group.

Abbreviations: $\mathrm{Cl}$, confidence interval; HR, hazard ratio; OS, overall survival; PFS, progression-free survival.

\section{Safety}

A total of 35 patients $(44.9 \%)$ across all treatment lines reported grade 3-4 AEs (Table 3); the most frequently reported of these were neutropenia (21.8\%), asthenia ( $8.9 \%)$, and hypertension (7.6\%). Bevacizumab treatment was discontinued in four patients due to AEs (severe epistaxis, digestive bleeding, brain stroke, and proteinuria).

\section{Discussion}

This retrospective analysis demonstrated that the combination of bevacizumab and paclitaxel was effective in a realworld setting in patients with HER2-negative MBC. The combination regimen achieved a high ORR (53.8\%). Longer PFS was seen in the first-line treatment setting compared with administration in second or subsequent lines. This decrease in efficacy was as expected, since treatment efficacy generally 
Table 3 Summary of grade 3/4 adverse events

\begin{tabular}{ll}
\hline Adverse event & Patients, $\mathbf{n}$ (\%) \\
\hline Grade 3/4 adverse events & $35(44.9)$ \\
Neutropenia & $17(21.8)$ \\
Asthenia & $7(8.9)$ \\
Hypertension & $6(7.6)$ \\
Febrile neutropenia & $4(5.1)$ \\
Proteinuria & $3(5.1)$ \\
Mucositis & $3(3.8)$ \\
Thrombocytopenia & $2(2.5)$ \\
Peripheral neuropathy & $2(2.5)$ \\
Bleeding & $2(2.5)$ \\
Thromboembolic disease & $1(1.2)$ \\
Brain stroke & $1(1.2)$ \\
\hline
\end{tabular}

declines with disease progression, due to such factors as increased tumor volume and drug resistance. Overall, these data are comparable with results obtained for first-line bevacizumab in combination with chemotherapy in randomized Phase III trials, with median PFS and ORR higher than those seen in the E2100 and ATHENA trials. ${ }^{1,8}$

The combination of bevacizumab and paclitaxel was also effective in both TNBC and hormone receptor-positive disease. Although bevacizumab and paclitaxel are effective in TNBC, patients with this type of MBC tend to have a worse prognosis than those with hormone receptor-positive disease, since TNBC has a more aggressive phenotype. In this study, there was no statistical difference in OS or PFS between TNBC and hormone receptor-positive disease, possibly due to the small sample size of TNBC patients. Although the size of the TNBC subgroup was too small to draw firm conclusions in this study, an analysis of the ATHENA trial also showed comparable OS in patients with hormone receptor-positive disease and TNBC when adjusted for prognostic factors. ${ }^{9}$

The combination regimen was generally well tolerated, with similar frequency and type of AEs (44.9\% with grade 3-4 AEs, 7.6\% hypertension, $5.1 \%$ proteinuria), as previously reported for bevacizumab plus taxane therapy. In the E2100 study, grade 3-4 hypertension occurred in $14.8 \%$ of patients and proteinuria in $3.5 \%$ of patients, ${ }^{1}$ whereas the AVADO trial reported grade $3-4$ AEs in $38.1 \%$ of patients, with hypertension in $4.5 \%$ and proteinuria in $2.0 \% .^{2}$ The ATHENA trial of first-line bevacizumab and taxane chemotherapy reported grade $\geq 3 \mathrm{AEs}$ in $51.5 \%$ of patients. ${ }^{8}$ The toxicity profile of bevacizumab is inherently linked to its mode of action; however, with adequate awareness and training, the side effects of bevacizumab-based regimens can be successfully managed without severe complications.
Various studies have investigated the prognostic and predictive factors of efficacy benefit in patients with MBC. Using an internally and externally validated score, Regierer et al reported DFI, location of metastases, and hormonereceptor status as significant prognostic factors for OS in $\mathrm{MBC} .{ }^{10}$ Prior anthracycline and taxane treatment and number of metastatic sites/liver disease have also been shown to be important prognostic factors in MBC. These factors were confirmed by multivariate analysis to be associated with worse OS in the ATHENA study. ${ }^{9}$ Analysis of these risk factors (DFI, liver metastases, previous anthracycline/taxane treatment, and TNBC) led to the differentiation of three prognostic groups with progressively worse prognosis: those with one or no risk factors, those with two risk factors, and those with three or four risk factors. ${ }^{9}$ These prognostic groups were seen in both TNBC and hormone receptor-positive MBC. Our results, showing that DFI $\leq 24$ months, three or more metastatic locations or liver metastases, and prior taxane therapy were associated with worse efficacy outcomes, are in agreement with these previous reports.

Patients receiving bevacizumab continuation had a longer PFS than those who did not. This may have been because most patients receiving maintenance therapy also responded to first-line bevacizumab plus paclitaxel. In addition, some patients in the group not receiving maintenance may have stopped receiving bevacizumab before disease progression, due to toxicity or the investigator's decision, which could also have contributed to the shorter PFS seen in this group. In a subgroup analysis of the ATHENA trial, patients who received bevacizumab maintenance therapy had longer PFS (11.6 months versus 6.7 months) and OS (30.0 months versus 18.4 months) than those who did not. ${ }^{11}$ Although the data may be biased because of the contributing factors surrounding patient selection for maintenance therapy (ie, inadvertently selecting responders), these results suggest that the use of maintenance bevacizumab continuation therapy could be beneficial for patients with MBC and warrants further investigation.

The addition of hormone therapy to bevacizumab continuation therapy in patients with estrogen receptor-positive disease is an important aspect of our analysis. Fabi et al reported a benefit of combination maintenance therapy, with patients receiving combined hormone therapy and bevacizumab maintenance therapy achieving a median PFS of 13.0 months compared with 4.1 months for patients treated with just bevacizumab maintenance therapy $(P=0.05) .{ }^{12}$ These results were supported by a Phase II trial of first-line hormone therapy plus bevacizumab that reported a median PFS of 
13.5 months. ${ }^{13}$ In the Phase III LEA study, the combination of hormone therapy and bevacizumab resulted in numerically but not significantly longer PFS compared with hormone therapy alone (19.3 versus 14.4 months, respectively). ${ }^{14}$ However, there are still limited conclusive data on whether bevacizumab plus hormone therapy as continuation therapy is superior to bevacizumab alone after stopping taxane-based therapy. In this retrospective analysis, patients with estrogen receptor-positive $\mathrm{MBC}$ who received bevacizumab plus hormone therapy had improved PFS and OS compared with patients who received bevacizumab monotherapy as continuation treatment, although the difference was not statistically significant because of the low patient numbers in these subgroups. The field of data remains inconclusive, and further research is warranted as to whether prescribing bevacizumab as continuation therapy alongside hormone therapy is the optimal treatment regimen in patients with HER2-negative MBC after weekly paclitaxel plus bevacizumab.

Overall, while our real-world findings are aligned with results of previous clinical studies, such as the E2100 trial, this analysis adds some important information about the possible benefits of this regimen in second and subsequent lines of therapy, as well as the hypothesis of an increased PFS with the addition of hormone therapy to bevacizumab continuation therapy. Due to the small sample size in this single-institution setting, some of our results did not reach statistical significance; however, the data are promising and warrant further investigation in a multicenter setting.

\section{Conclusion}

The combination of bevacizumab plus paclitaxel was active in patients with $\mathrm{MBC}$, as well as in subgroups of patients with TNBC and those with hormone receptor-positive disease, as observed in a real-world setting. Continuation of bevacizumab after stopping paclitaxel, and the addition of hormone therapy to bevacizumab, in patients with estrogen receptor-positive $\mathrm{MBC}$ could be beneficial, as shown by the longer PFS and OS observed. Additional research is needed to further confirm the use of bevacizumab and hormone therapy as continuation therapy for MBC.

\section{Acknowledgments}

Third-party medical writing assistance for this manuscript was provided by Joanna Musgrove of Gardiner-Caldwell Communications, funded by Fritz Hoffmann-La Roche.

\section{Disclosure}

The authors report no conflicts of interest in this work.

\section{References}

1. Miller K, Wang M, Gralow J, et al. Paclitaxel plus bevacizumab vs paclitaxel alone for metastatic breast cancer. $N$ Engl J Med. 2007;357:2666-2676.

2. Miles DW, Chan A, Dirix LY, et al. Phase III study of bevacizumab plus docetaxel compared with placebo plus docetaxel for the first-line treatment of human epidermal growth factor receptor 2-negative metastatic breast cancer. J Clin Oncol. 2010;28:3239-3247.

3. Robert NJ, Diéras V, Glaspy J, et al. RIBBON-1: randomized, double-blind, placebo-controlled, phase III trial of chemotherapy with or without bevacizumab for first-line treatment of human epidermal growth factor receptor 2-negative, locally recurrent or metastatic breast cancer. J Clin Oncol. 2011;29:1252-1260.

4. Liu Y, Tamimi RM, Collins LC, et al. The association between vascular endothelial growth factor expression in invasive breast cancer and survival varies with intrinsic subtypes and use of adjuvant systemic therapy: results from the Nurses' Health Study. Breast Cancer Res Treat. 2011;129:175-184.

5. Fakhrejahani E, Toi M. Tumor angiogenesis: pericytes and maturation are not to be ignored. J Oncol. 2012;2012:261750.

6. Montero AJ, Escobar M, Lopes G, Glück S, Vogel C. Bevacizumab in the treatment of metastatic breast cancer: friend or foe? Curr Oncol Rep. 2012;14:1-11.

7. Brufsky A, Valero V, Tiangco B, et al. Second-line bevacizumabcontaining therapy in patients with triple-negative breast cancer: subgroup analysis of the RIBBON-2 trial. Breast Cancer Res Treat. 2012;133:1067-1075.

8. Smith IE, Pierga JY, Biganzoli L, et al. First-line bevacizumab plus taxane-based chemotherapy for locally recurrent or metastatic breast cancer: safety and efficacy in an open-label study in 2,251 patients. Ann Oncol. 2011;22:595-602.

9. Llombart-Cussac A, Pivot XB, Biganzoli L, et al. A prognostic factor (PF) index for overall survival in a HER2-negative endocrine-resistant metastatic breast cancer (MBC) population: analysis from the ATHENA trial. J Clin Oncol. 2013;31:555.

10. Regierer AC, Wolters R, Ufen MP, et al. An internally and externally validated prognostic score for metastatic breast cancer: analysis of 2269 patients. Ann Oncol. 2014;25:633-638.

11. Smith I, Pierga JY, Biganzoli L, et al. Final overall survival results and effect of prolonged ( $\geq 1$ year) first-line bevacizumab-containing therapy for metastatic breast cancer in the ATHENA trial. Breast Cancer Res Treat. 2011;130:133-143.

12. Fabi A, Russillo M, Ferretti G, et al. Maintenance bevacizumab beyond first-line paclitaxel plus bevacizumab in patients with Her2-negative hormone receptor-positive metastatic breast cancer: efficacy in combination with hormonal therapy. BMC Cancer. 2012;12:482.

13. Yardley DA, Burris HA 3rd, Clark BL, et al. Hormonal therapy plus bevacizumab in postmenopausal patients who have hormone receptorpositive metastatic breast cancer: a phase II trial of the Sarah Cannon Oncology Research Consortium. Clin Breast Cancer. 2011;11: 146-152.

14. Loibl S, De la Haba J, von Minckwitz G, et al. Phase III trial evaluating the addition of bevacizumab to endocrine therapy as first-line treatment for advanced breast cancer. Final analysis of the LEA study. Poster presented at: 2013 European Cancer Congress; September 27-October 1, 2013; Amsterdam. 
OncoTargets and Therapy

\section{Publish your work in this journal}

OncoTargets and Therapy is an international, peer-reviewed, open access journal focusing on the pathological basis of all cancers, potential targets for therapy and treatment protocols employed to improve the management of cancer patients. The journal also focuses on the impact of management programs and new therapeutic agents and

Submit your manuscript here: http://www.dovepress.com/oncotargets-and-therapy-journal
Dovepress

protocols on patient perspectives such as quality of life, adherence and satisfaction. The manuscript management system is completely online and includes a very quick and fair peer-review system, which is all easy to use. Visit http://www.dovepress.com/testimonials.php to read real quotes from published authors. 